\title{
Texture Enhancement for Medical Images Based on Fractional Differential Masks
}

\author{
Hamid A. Jalab ${ }^{1}$ and Rabha W. Ibrahim ${ }^{2}$ \\ ${ }^{1}$ Faculty of Computer Science and Information Technology, University of Malaya, 50603 Kuala Lumpur, Malaysia \\ ${ }^{2}$ Institute of Mathematical Sciences, University of Malaya, 50603 Kuala Lumpur, Malaysia
}

Correspondence should be addressed to Rabha W. Ibrahim; rabhaibrahim@yahoo.com

Received 13 January 2013; Revised 6 March 2013; Accepted 6 March 2013

Academic Editor: Thabet Abdeljawad

Copyright (C) 2013 H. A. Jalab and R. W. Ibrahim. This is an open access article distributed under the Creative Commons Attribution License, which permits unrestricted use, distribution, and reproduction in any medium, provided the original work is properly cited.

\begin{abstract}
Texture enhancement for medical images is the most important technique in medical image diagnosis. This paper introduces a texture enhancement technique for medical images by using fractional differential (FD) masks based on Srivastava-Owa fractional operators. We also construct a 2D isotropic gradient mask based on generalized fractional operators. Texture enhancement performance is measured by applying experiments according to visual perception and by using Sobel/Canny edge filters and graylevel co-occurrence matrix. We discuss the capability of the FD mask for texture enhancement. The experiments and analysis show that the operator can extract subtle information and make the edges prominent.
\end{abstract}

\section{Introduction}

Fractional calculus and its applications (e.g., the theory of derivatives and integrals of any arbitrary, real, or complex order) are significant for different areas of mathematics, physics, and engineering. Fractional calculus generalizes the ideas of integer-order differentiation and $n$-fold integration. Fractional derivatives introduce an excellent instrument for describing the general properties of various materials and processes. This feature is the main advantage of fractional derivatives compared with classical integer-order models, in which such effects are neglected. The advantages of fractional derivatives become apparent in modeling, describing the mechanical, electrical, and physical properties of real materials [1-4], and removing noise and irrelevant information from the absorption spectra [5].

Studies on fractional calculus and fractional differential (FD) equations that involve different operators, such as Riemann-Liouville operators, Erd'elyi-Kober operators, Weyl-Riesz operators, Caputo operators, and GrünwaldLetnikov operators, have evolved during the past four decades and have been extended in other fields [6].

Fractional calculus (integral and differential operators) has been employed recently in signal and image possessing.
Fractional calculations are capable of enhancing image quality for revealing faint objects in astronomical image analysis $[7,8]$ and signal analysis [9]; this approach provides interesting possibilities for edge detection and image restoration. FD has received considerable attention in various image enhancement applications such as texture enhancement [10] and image denoising [6].

Medical images are a key investigation tool in medical diagnosis. The quality of a medical image is determined by the imaging method, equipment characteristics, and imaging variables selected by the operator. A large number of medical image acquisition devices exist such as computed tomography scanners, magnetic resonance imagers, ultrasound probes, and nuclear imagers [11, 12].

Medical images usually have low resolutions, high noise, and high textures. Moreover, medical images represent an enormous amount of data. The noise in medical images renders the interpretation of images difficult. Thus, denoising is necessary before the analysis of medical images. Denoising techniques have been exploited in the field of medicine $[13,14]$.

Texture is an important feature of natural images. Thus, various image texture applications have been subjected to intense studies by researchers [15]. Image texture is defined 
as the function of the spatial variation in pixel intensities (gray values). Thus, image texture is one of the fundamental factors required in medical imaging analysis. Castellano et al. [16] clarified the principles of texture analysis in medical images and concluded that texture parameters are simply mathematical representations of image features, which can be categorized as smooth, rough, grainy, and so on. This result implies that texture analysis can be applied to any set of image regions that can be differentiated by such descriptions. Smith and Chang [17] defined texture as a visual pattern with homogenous properties that cannot result from the presence of only a single color or intensity. Therefore, texture parameter analysis is a useful method of increasing obtainable information from medical images.

Texture enhancement is one of the most important techniques in medical image processing. The most common method for enhancing texture is by using masks (also referred to as operators, filters, or templates), which is basically a small $2 \mathrm{D}$ array. Each pixel is modified in the mask according to the value of the neighborhood around the pixel of interest. The texture enhancement technique based on mask operations has become increasingly important in image processing and analysis [18].

The frequencies of an image determine the changes in gray values with changing distance. High-frequency components are characterized by large changes in gray values over small distances; edges and noise are examples of highfrequency components. By contrast low-frequency components are characterized by small changes in gray values; backgrounds and textures are examples of low-frequency components [19].

The FD mask preserves low-frequency contour features in smooth areas, nonlinearly maintains high-frequency marginal features in areas wherein the changes in gray level are considerable, and enhances texture details in areas wherein the changes in gray level are insignificant [17].

Several works have used mask-based texture enhancement by using FD. Qing-li et al. [20] constructed an 2D isotropic nonlinear filter algorithm based on a FD algorithm for image contrast enhancement. The structure and parameters of the algorithm were presented in eight directions, and fractional-order differential operators were used to control the image contrast enhancement. This nonlinear filter algorithm was implemented on various low-contrast digital images.

Yang et al. [21] showed that image edge detection, which is based on FD operators and not traditional first and second differential operators, has a higher signal-to-noise ratio. This work was a preliminary attempt in exploring fractional-order differential operators in image processing applications. Garg and Singh [22] proposed a 2D FD operator, which is an improved version of the Grümwald-Letnikov FD operator. The improved Grümwald-Letnikov FD operator enhances both the texture and brightness of the image. $\mathrm{Pu}$ et al. [23] proposed six FD masks. They demonstrated the most efficient fractional mask by theoretic and experimental analysis. Their experiments show that the FD-based approach is better than traditional integral differential-based algorithms when nonlinearly enhancing comprehensive texture details in the smooth areas of texture-rich digital images.

In our previous work, we proposed a generalized FD based on the generalized Savitzky-Golay filtering of Srivastava-Owa fractional operators for image texture enhancement. This algorithm was used to control the degree of texture enhancement with fractional power values.

We aim to develop a differential mask based on Srivastava-Owa fractional operators for the texture enhancement of grayscale medical images and demonstrate the capability of the FD mask by varying the FD power parameters.

This paper is organized as follows. Section 2 explains the discrete FD mask. Section 3 presents the simulation results. Finally, Section 4 concludes.

\section{Discreet FD Mask}

This section describes the mathematical background of the differential operator mask in the proposed algorithm. We constructed a generalized fractional mask by using the following generalized FD operator [24]:

$$
\begin{array}{r}
D_{Z}^{\alpha, \mu} f(z):=\frac{(\mu+1)^{\alpha}}{\Gamma(1-\alpha)} \frac{d}{d z} \int_{0}^{z} \frac{\zeta^{\mu} f(\zeta)}{\left(z^{\mu+1}-\zeta^{\mu+1}\right)^{\alpha}} d \zeta \\
0<\alpha \leq 1,
\end{array}
$$

where the function $f(z)$ is analytic in simply connected region of the complex $z$-plane $\mathscr{C}$ that contains the origin. The multiplicity of $\left(z^{\mu+1}-\zeta^{\mu+1}\right)^{-\alpha}$ is removed by requiring $\log \left(z^{\mu+1}-\zeta^{\mu+1}\right)$ to be real when $\left(z^{\mu+1}-\zeta^{\mu+1}\right)>0$.

We can compute the value of the FD operator in (1) by using the numerical calculation that references the discrete form. If the interval is $[0, z], z$ is real, $n$ is divided into equal parts, and the differential of each part is calculated and combined. Thus, we observe the following:

$$
\begin{aligned}
D_{Z}^{\alpha, \mu} f(z)= & \frac{(\mu+1)^{\alpha}}{\Gamma(1-\alpha+m)}\left(\frac{d}{d z}\right)^{m+1} \\
& \times \int_{0}^{z} \zeta^{\mu} f(\zeta)\left(z^{\mu+1}-\zeta^{\mu+1}\right)^{m-\alpha} d \zeta \\
= & \frac{(\mu+1)^{\alpha}}{\Gamma(1-\alpha+m)} \sum_{j=1}^{n}\left(\frac{d}{d z}\right)^{m+1} \\
& \times j^{\mu} f(j)\left(z^{\mu+1}-j^{\mu+1}\right)^{m-\alpha} \\
= & \frac{(\mu+1)^{\alpha}}{\Gamma(1-\alpha+m)} \sum_{j=1}^{n} j^{\mu} f(j) \\
& \times[(m-\alpha) \times(m-\alpha-1) \times \cdots \times(-\alpha) \\
& \quad \times(\mu+1)^{m+1}\left(z^{\mu+1}-j^{\mu+1}\right)^{-\alpha-1} z^{(m+1) \mu-0} \\
& \quad+(m-\alpha) \times(m-\alpha-1) \times \cdots \times(1-\alpha) \\
& \quad \times(\mu+1)^{m}(m+1) \mu\left(z^{\mu+1}-j^{\mu+1}\right)^{-\alpha} z^{m \mu-1} \\
& \quad+(m-\alpha) \times(m-\alpha-1) \times \cdots \times(2-\alpha)
\end{aligned}
$$




\begin{tabular}{|c|c|c|c|c|}
\hline 0 & 0 & 0 & 0 & 0 \\
\hline 0 & 0 & 0 & 0 & 0 \\
\hline$\varphi_{n-1}$ & $\cdots$ & $\varphi_{2}$ & $\varphi_{1}$ & $\varphi_{0}$ \\
\hline 0 & 0 & 0 & 0 & 0 \\
\hline 0 & 0 & 0 & 0 & 0 \\
\hline
\end{tabular}

(a)

\begin{tabular}{|c|c|c|c|c|}
\hline 0 & 0 & 0 & 0 & 0 \\
\hline 0 & 0 & 0 & 0 & 0 \\
\hline$\varphi_{0}$ & $\varphi_{1}$ & $\varphi_{2}$ & $\cdots$ & $\varphi_{n-1}$ \\
\hline 0 & 0 & 0 & 0 & 0 \\
\hline 0 & 0 & 0 & 0 & 0 \\
\hline
\end{tabular}

(c)

\begin{tabular}{|c|c|c|c|c|}
\hline 0 & 0 & 0 & 0 & $\varphi_{n-1}$ \\
\hline 0 & 0 & 0 & $\cdots$ & 0 \\
\hline 0 & 0 & $\varphi_{2}$ & 0 & 0 \\
\hline 0 & $\varphi_{1}$ & 0 & 0 & 0 \\
\hline$\varphi_{0}$ & 0 & 0 & 0 & 0 \\
\hline
\end{tabular}

(e)

\begin{tabular}{|c|c|c|c|c|}
\hline$\varphi_{0}$ & 0 & 0 & 0 & 0 \\
\hline 0 & $\varphi_{1}$ & 0 & 0 & 0 \\
\hline 0 & 0 & $\varphi_{2}$ & 0 & 0 \\
\hline 0 & 0 & 0 & $\cdots$ & 0 \\
\hline 0 & 0 & 0 & 0 & $\varphi_{n-1}$ \\
\hline
\end{tabular}

(g)

\begin{tabular}{|c|c|c|c|c|}
\hline 0 & 0 & $\varphi_{n-1}$ & 0 & 0 \\
\hline 0 & 0 & $\vdots$ & 0 & 0 \\
\hline 0 & 0 & $\varphi_{2}$ & 0 & 0 \\
\hline 0 & 0 & $\varphi_{1}$ & 0 & 0 \\
\hline 0 & 0 & $\varphi_{0}$ & 0 & 0 \\
\hline
\end{tabular}

(b)

\begin{tabular}{|c|c|c|c|c|}
\hline 0 & 0 & $\varphi_{0}$ & 0 & 0 \\
\hline 0 & 0 & $\varphi_{1}$ & 0 & 0 \\
\hline 0 & 0 & $\varphi_{2}$ & 0 & 0 \\
\hline 0 & 0 & $\vdots$ & 0 & 0 \\
\hline 0 & 0 & $\varphi_{n-1}$ & 0 & 0 \\
\hline
\end{tabular}

(d)

\begin{tabular}{|c|c|c|c|c|}
\hline$\varphi_{n-1}$ & 0 & 0 & 0 & 0 \\
\hline 0 & $\cdots$ & 0 & 0 & 0 \\
\hline 0 & 0 & $\varphi_{2}$ & 0 & 0 \\
\hline 0 & 0 & 0 & $\varphi_{1}$ & 0 \\
\hline 0 & 0 & 0 & 0 & $\varphi_{0}$ \\
\hline
\end{tabular}

(f)

\begin{tabular}{|c|c|c|c|c|}
\hline 0 & 0 & 0 & 0 & $\varphi_{0}$ \\
\hline 0 & 0 & 0 & $\varphi_{1}$ & 0 \\
\hline 0 & 0 & $\varphi_{2}$ & 0 & 0 \\
\hline 0 & $\cdots$ & 0 & 0 & 0 \\
\hline$\varphi_{n-1}$ & 0 & 0 & 0 & 0 \\
\hline
\end{tabular}

(h)

FIGURE 1: Fractional masks at $180^{\circ}, 90^{\circ}, 0^{\circ}, 270^{\circ}, 45^{\circ}, 135^{\circ}, 315^{\circ}$, and $225^{\circ}$.

$$
\begin{aligned}
& \times(\mu+1)^{m-1}(m-1) \\
& \left.\times \mu(\mu-1)\left(z^{\mu+1}-j^{\mu+1}\right)^{1-\alpha} z^{(m-1) \mu-2}+\cdots\right]
\end{aligned}
$$

However, an image is a function of two variables; thus, we can generalize these definitions to include both the $x$ and $y$ values. If the image is required to complete the nonlinear filter, we must convolute the pixel values of the image with a $(s \times t)$ mask:

$$
h(x, y)=\sum_{s=-a}^{a} \sum_{t=-b}^{b} w(s, t) f(x+s, y+t),
$$

where $t=n, f$ is the pixel value, and $w$ is the value of the mask. The mask is designed into $r \times r$ size matrix $M$, which 


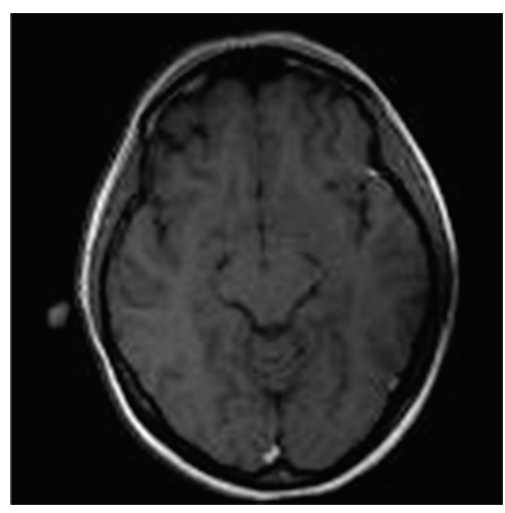

Original image

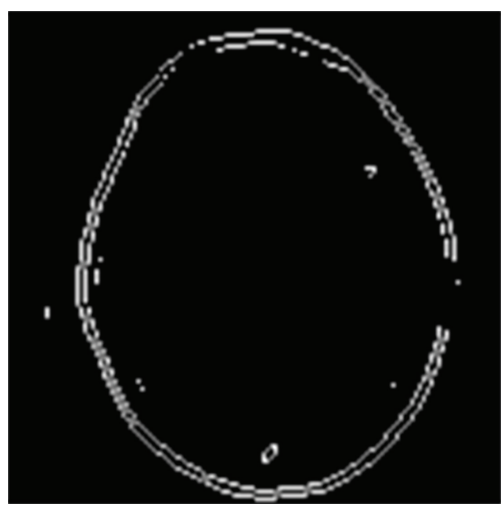

Sobel for original image

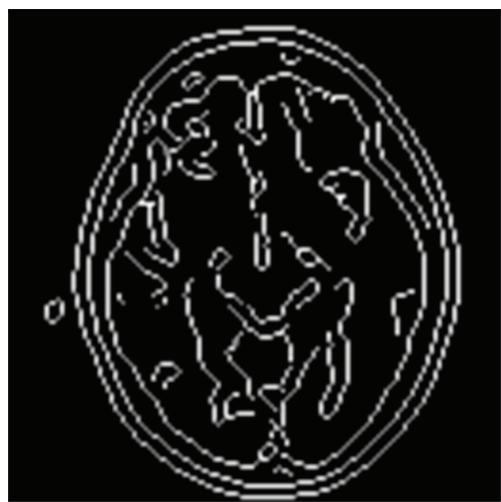

Canny for original image

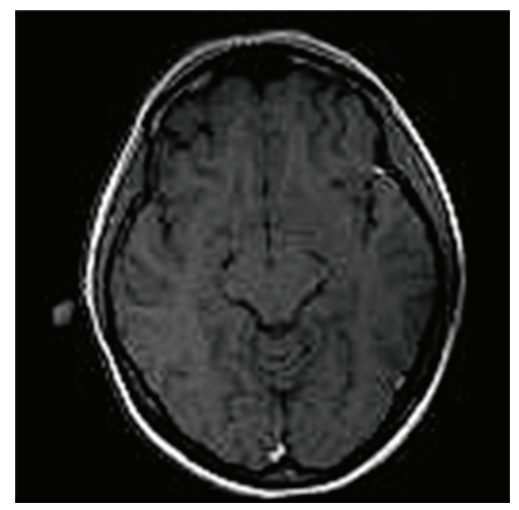

FD enhanced image

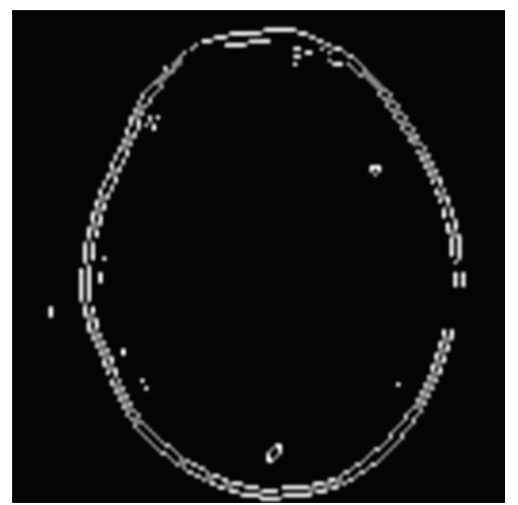

Sobel for FD enhanced image

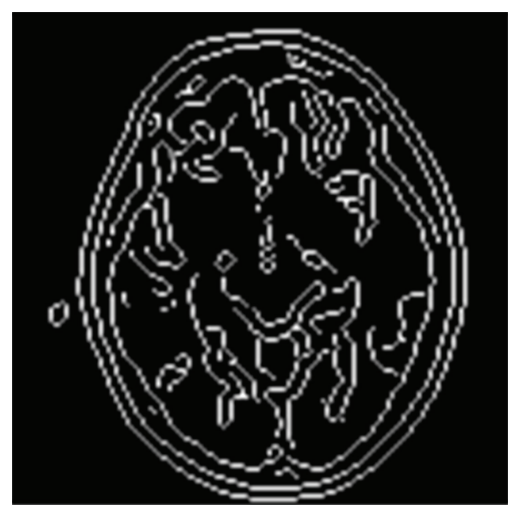

Canny for FD enhanced image

FIGURE 2: Results of the proposed enhancement algorithm by using FD masks for $\rho=6, \mu=0.05$, and $\alpha=0.1$.

has $r$ layers ( $r$ is odd). $M$ has eight directions: $0^{\circ}, 45^{\circ}, 90^{\circ}, 135^{\circ}$, $180^{\circ}, 225^{\circ}, 270^{\circ}$, and $315^{\circ}$ (Figure 1). The distinct $j$ th layer of $M$ is $2 \times j$. From the above construction, we conclude the following:

$$
\begin{aligned}
M_{j} \cong & \frac{(\mu+1)^{\alpha}}{\Gamma(1-\alpha+m)} \\
& \times[(m-\alpha) \times(m-\alpha-1) \\
& \quad \times \cdots \times(-\alpha)(\mu+1)^{m+1}(2 \times j)^{-\alpha-1} \\
& \quad+(m-\alpha) \times(m-\alpha-1) \times \cdots \times(1-\alpha)
\end{aligned}
$$

$$
\begin{aligned}
& \times(\mu+1)^{m}(m+1) \mu(2 \times j)^{-\alpha}+(m-\alpha) \\
& \times(m-\alpha-1) \times \cdots \times(2-\alpha) \\
& \left.\times(\mu+1)^{m-1}(m-1) \mu(\mu-1)(2 \times j)^{1-\alpha}+\cdots\right] .
\end{aligned}
$$

If we assume that $m=0, \alpha \in(0,1)$, and $\mu \geq 0$, we obtain the following:

$$
M_{j}=\frac{(\mu+1)^{\alpha+1}}{\Gamma(1-\alpha)}(-\alpha)(2 \times j)^{-\alpha-1}
$$




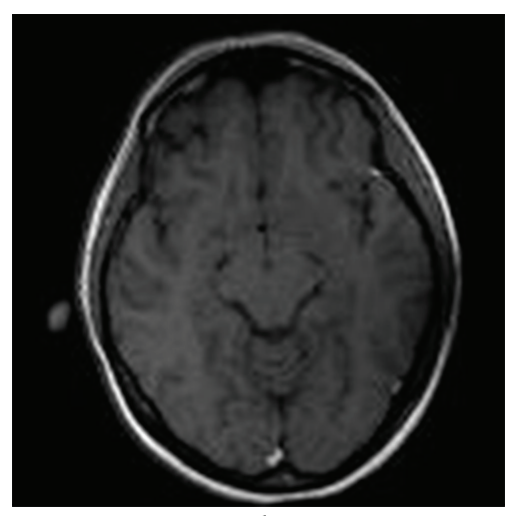

Original image

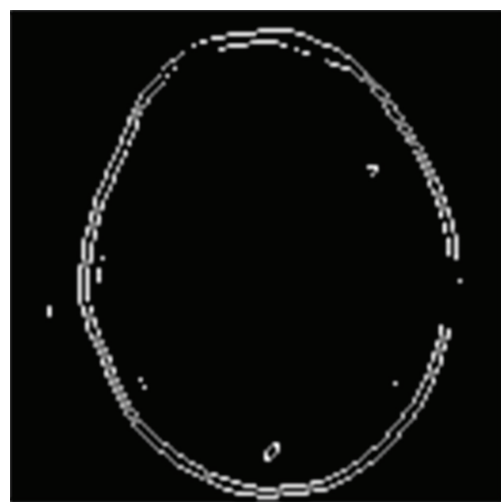

Sobel for original image

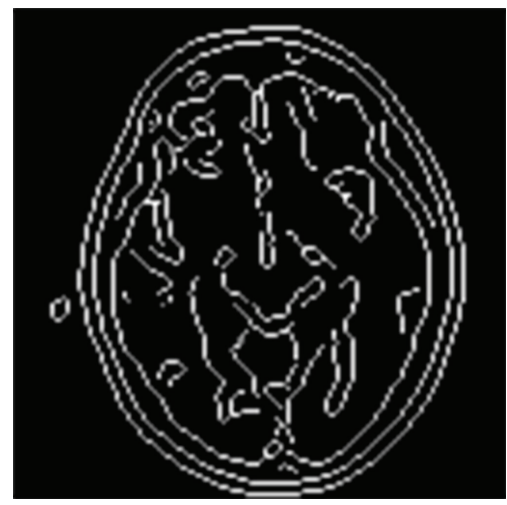

Canny for original image

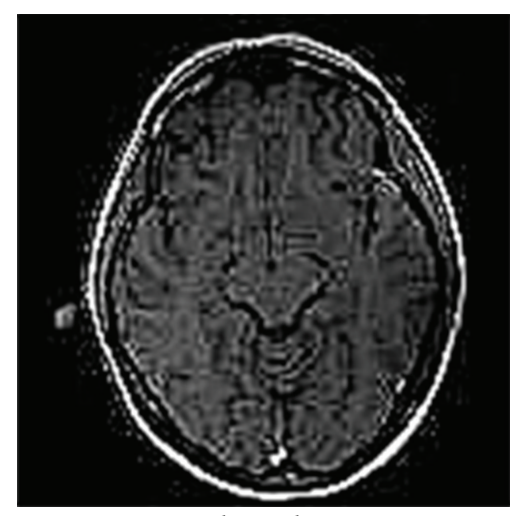

FD enhanced image

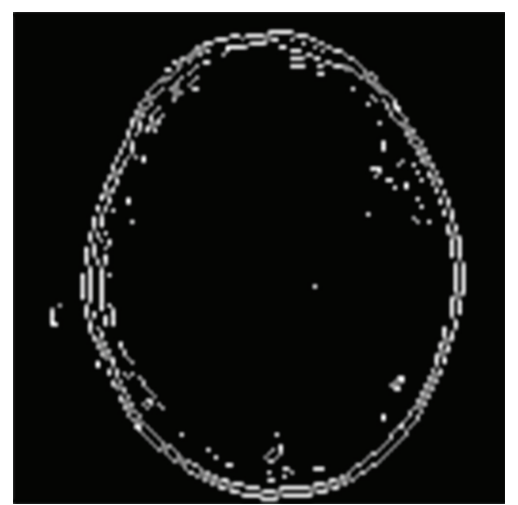

Sobel for FD enhanced image

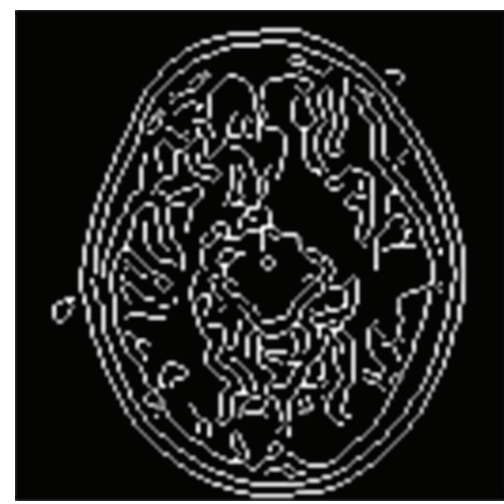

Canny for FD enhanced image

FIGURE 3: Results of the proposed enhancement algorithm by using FD masks for $\rho=6, \mu=0.05$, and $\alpha=0.5$.

Specifically, when $\mu=0$, the case of the Riemann-Liouville fractional operator is obtained:

$$
M_{j}=\frac{1}{\Gamma(1-\alpha)}(-\alpha)(2 \times j)^{-\alpha-1} .
$$

For texture enhancement, the sharpening edges must add to the value of the ordinal pixel. Thus, we impose that $\phi_{j}=\rho M_{j}$, where $\rho>0$ is the intensity factor:

$$
\begin{gathered}
\phi_{j}=\frac{\rho}{\Gamma(1-\alpha)}(-\alpha)(2 \times j)^{-\alpha-1}, \\
\phi_{j}=\frac{\rho(\mu+1)^{\alpha+1}}{\Gamma(1-\alpha)}(-\alpha)(2 \times j)^{-\alpha-1},
\end{gathered}
$$

$$
\begin{aligned}
& \phi_{j}=\frac{\rho(}{\Gamma+1)^{\alpha}} \\
& \times[(m-\alpha) \times(m-\alpha-1) \times \cdots \\
& \times(-\alpha)(\mu+1)^{m+1}(2 \times j)^{-\alpha-1} \\
&+(m-\alpha) \times(m-\alpha-1) \times \cdots \\
& \times(1-\alpha)(\mu+1)^{m}(m+1) \mu(2 \times j)^{-\alpha} \\
&+(m-\alpha) \times(m-\alpha-1) \times \cdots \\
& \times(2-\alpha)(\mu+1)^{m-1}(m-1) \mu(\mu-1)(2 \times j)^{1-\alpha} \\
&+\cdots]
\end{aligned}
$$




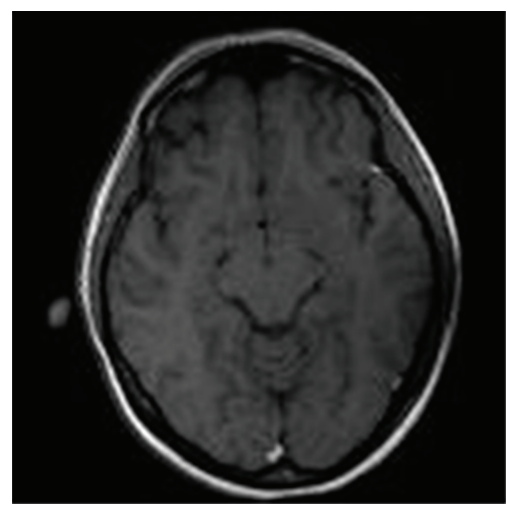

Original image

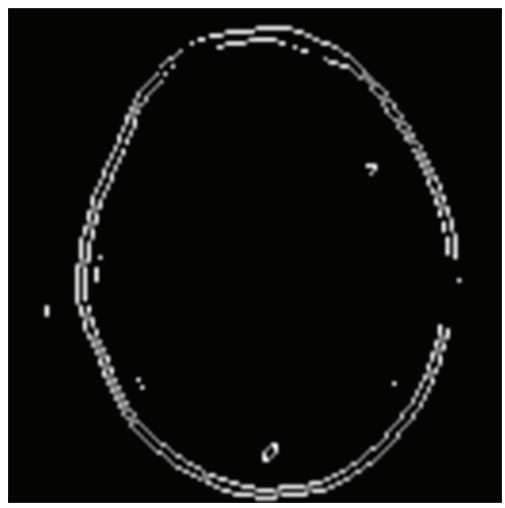

Sobel for original image

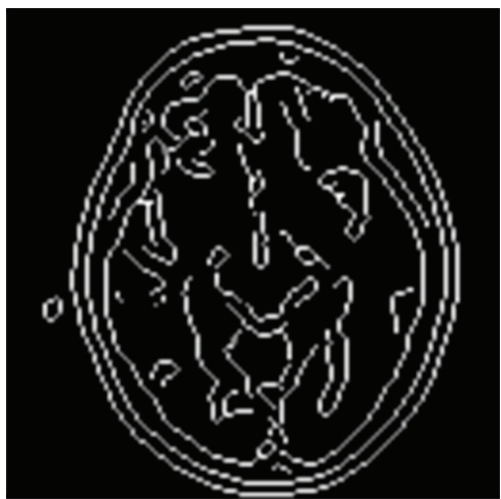

Canny for original image

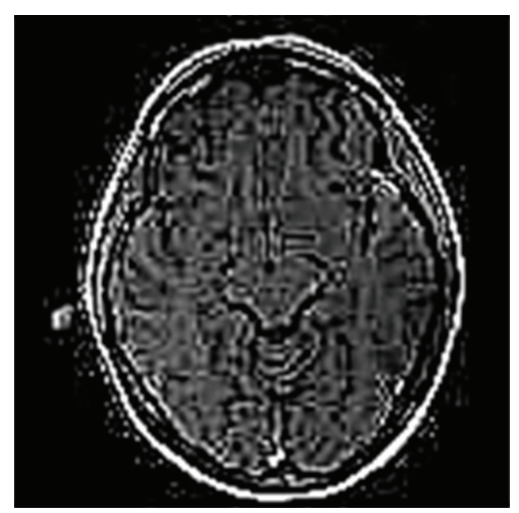

FD enhanced image

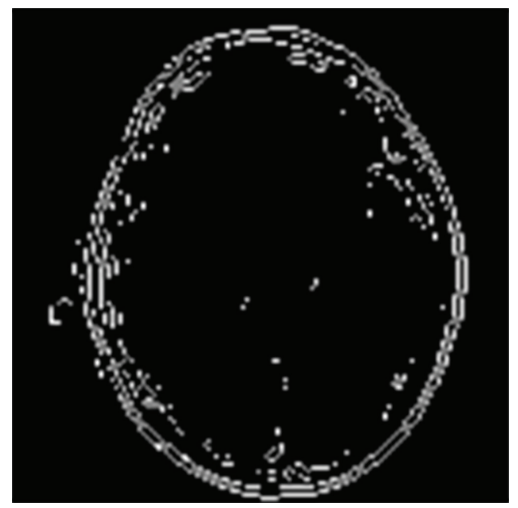

Sobel for FD enhanced image

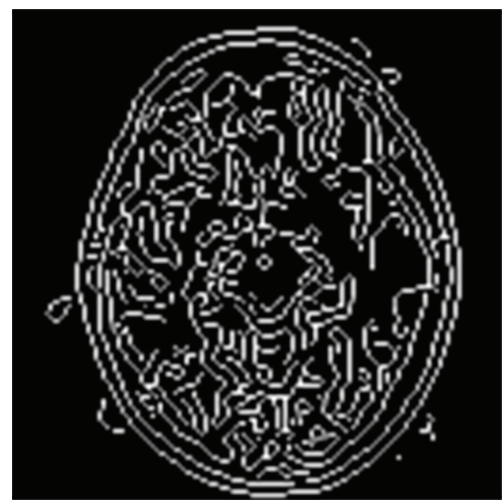

Canny for FD enhanced image

FIGURE 4: Results of the proposed enhancement algorithm by using FD masks for $\rho=6, \mu=0.05$, and $\alpha=0.9$.

For digital images, the coefficients of 2D FD masks can be obtained in eight directions: $180^{\circ}, 90^{\circ}, 0^{\circ}, 270^{\circ}, 45^{\circ}$, $135^{\circ}, 315^{\circ}$, and $225^{\circ}$ (Figure 1 ). These masks are located on the negative $y$-coordinate, negative $x$-coordinate, positive $y$-coordinate, positive $x$-coordinate, left upward diagonal, right upward diagonal, left downward diagonal, and right downward diagonal, respectively. These masks are rotation invariant and are used to describe the textures and edges in images.

The values of $\varphi_{j}$ are provided in (7), and $\varphi_{0}$ is calculated as follows [25]:

$$
\phi_{0}=1-\sum_{j=1}^{n}(8 j) \phi_{j} .
$$

The nine values of each FD mask are obtained by sliding the mask window over the image from the top left corner of the image block through all pixels until the FD mask fits entirely within the image boundaries. The output of each image block is eight values, which represents the texture information of each image. The dimension of the FD mask is an odd natural number. To obtain a good result, the size of the mask should be small.

\section{Experimental Results and Discussion}

This section demonstrates the texture-enhancing capability of the 2D FD discrete gradient operator based on SrivastavaOwa fractional operators. We analyze the texture-enhancing 


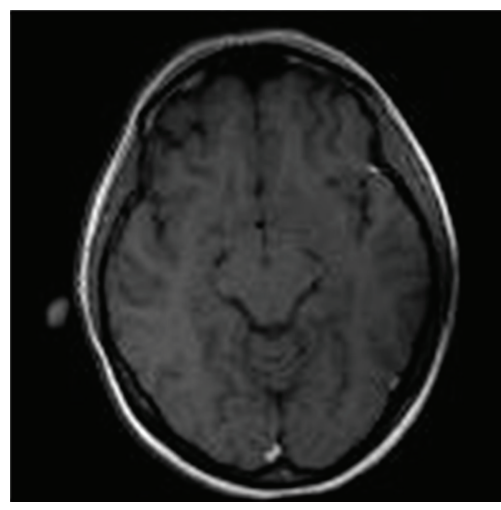

Original image

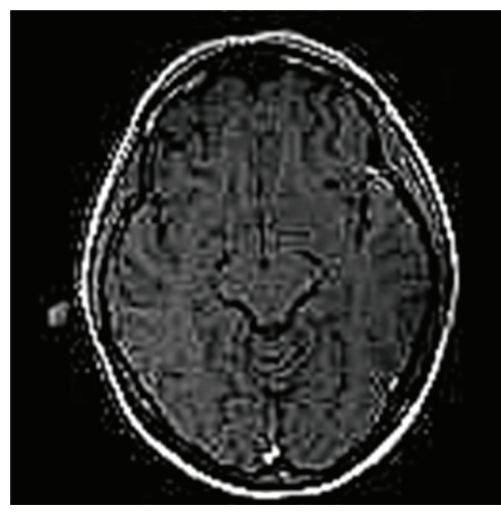

FD enhanced image with $\alpha=0.5$

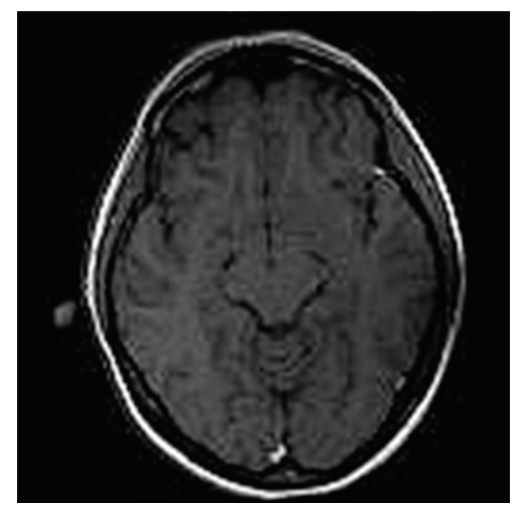

FD enhanced image with $\alpha=0.1$

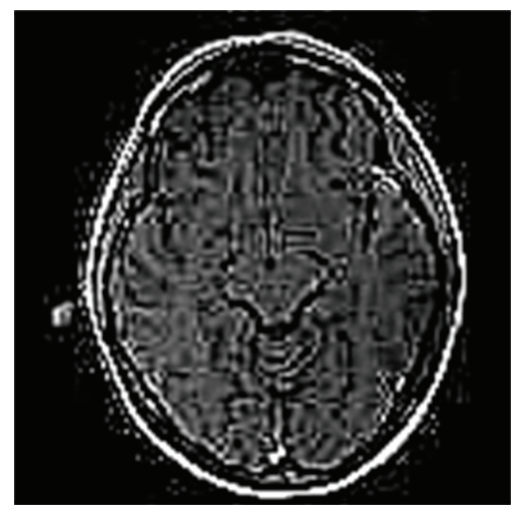

FD enhanced image with $\alpha=0.9$

FIGURE 5: Summary of the results of the proposed enhancement for $\alpha=0.1,0.5$, and 0.9 in the Riemann-Liouville case where $\mu=0$.

capability of the FD masks based on visual perception and discuss the relationship between fractional power parameters $(\alpha$ and $\mu)$ and texture-enhancing details by using Sobel/Canny edge filters and gray-level co-occurrence matrix (GLCM). We then discuss the texture-enhancing capability of the FD masks and compare them with those of traditional approaches.

Performance tests for the proposed algorithm are implemented by using Matlab 2011a on an Intel Core i7 $2.2 \mathrm{GHz}$ computer with 4 GB DDR3 RAM and 64-bit Window 7 OS.

The values of the fractional power are $\alpha$ is lower than one, $\mu$ is greater than zero, and parameter $\rho$ is used to control the degree of enhancement; the value of $\rho$ is $\rho \in R$. The proposed algorithm not only enhances the texture quality of the image but also maintains the edges present in the image.

The proposed texture enhancement algorithm includes the following steps.

(i) Read the original medical grayscale image.

(ii) Set the mask window size and the values of the fractional power parameters, $\alpha$ and $\mu$.

(iii) Apply FD masks on all eight directions with the gray value of the corresponding image pixels.

(iv) Obtain the eight values of each fractional differential mask by sliding the mask window over the image. (v) Apply Sobel/Canny edge filters and GLCM to both the original and enhanced images.

(vi) Repeat Steps (ii) to (v) for different fractional power values.

By varying $\alpha$ and keeping both $\mu=0.05$ and $\rho=6$ constant, variations in image texture are observed. The results are compared by using Sobel/Canny edge methods and GLCM.

The algorithm for digital color images is similar to that for gray images but performs separately for each of the RGB components.

The Sobel method finds edges by using Sobel approximation on the derivative. The Sobel method returns edges at the points where the image achieves a maximum gradient. The Canny method finds edges by determining the local maxima of the gradient of the image. The gradient is calculated by using the derivative of a Gaussian filter [26].

One of the most traditional ways to analyze the spatial distribution of the gray levels of an image is by using GLCM. The following parameters are used frequently in the literature: contrast, correlation, energy, and homogeneity [27].

From a human visual perspective, the proposed enhancement algorithm with FD masks has good enhancement performance for both testing images under different degrees of fractional power values (Figures 2, 3, and 4). The increasing value of $\alpha(0.1,0.5$, and 0.9$)$ increases the number of pixels, thus improving the contrast and clarity of the image. 


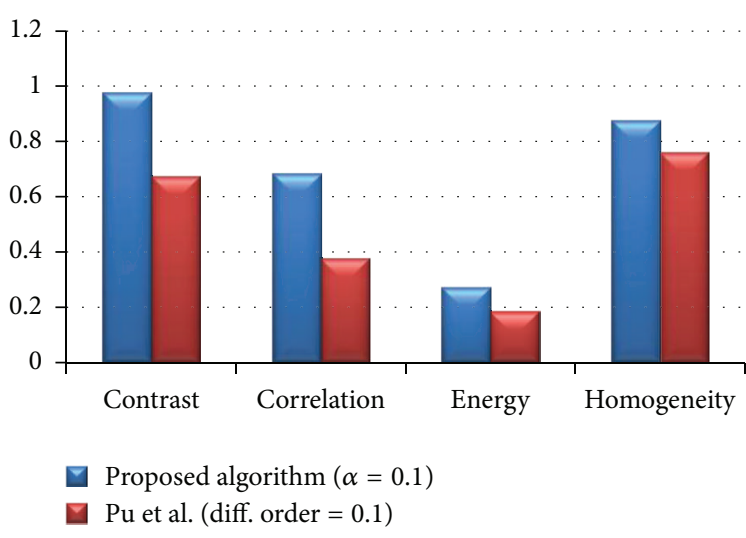

(a)

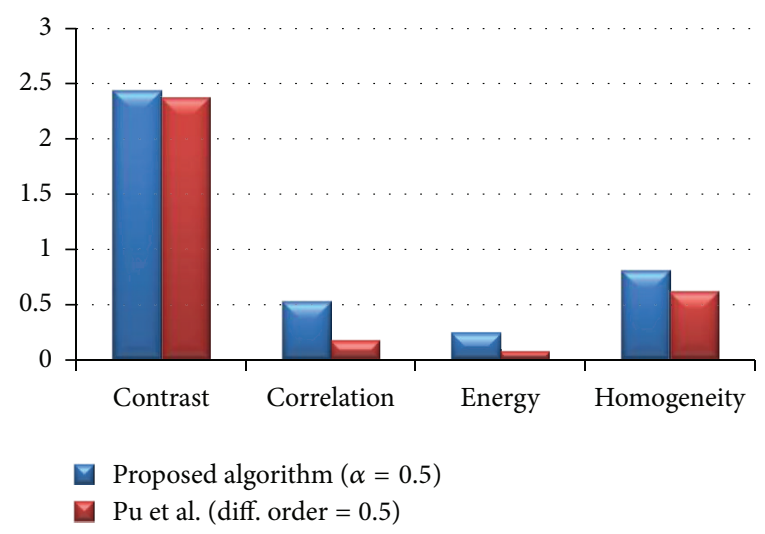

(b)

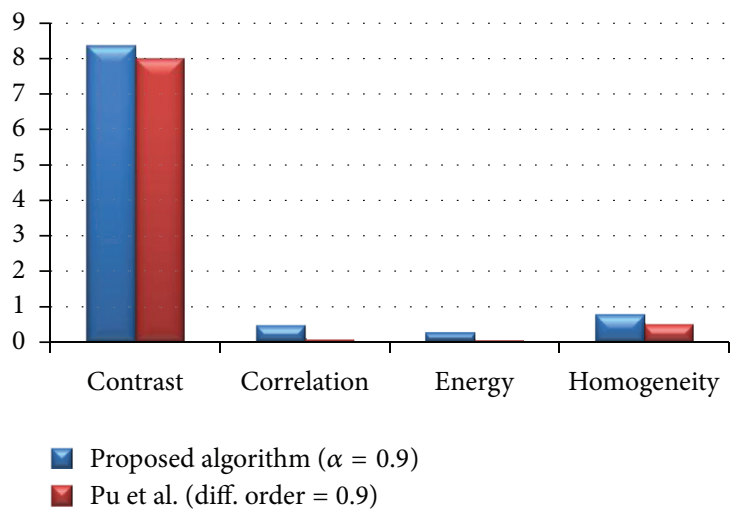

(c)

FIGURE 6: Comparison of texture enhancement with other works for different values of $\alpha$ : (a) $\alpha=0.1$, (b) $\alpha=0.5$, and (c) $\alpha=0.9$.

Figure 5 shows the summary of the results of the proposed enhancement for different values of $\alpha$ and Riemann-Liouville cases where $\mu=0$. By using qualitative analysis, we observe that the first image with $\alpha=0.9$ is clearer than all images, even for the Riemann-Liouville case where $\mu=0$. The proposed algorithm not only enhances the texture quality of the image but also maintains the edges present in the image. The proposed algorithm for image texture enhancement provides satisfactory results. The qualitative analysis of the proposed algorithm serves as one of the most important parameters in judging performance.

For the quantity analysis, we use GLCM to describe the comprehensive information of the texture details. Figure 6 compares the texture enhancement of the present study with that of $\mathrm{Pu}$ et al. [23] for $\alpha=0.1,0.5$, and 0.9. Pu et al. [23] developed six FD masks based on Grümwald-Letnikov operators and presented the structures and parameters of each mask in eight directions. The main differences between the present study and the study of Pu et al. [23] are as follows.

(1) The FD masks of $\mathrm{Pu}$ et al. [23] are based on Grümwald-Letnikov operators. In the present study, texture enhancement is introduced by employing a generalized 2D FD discrete gradient operator based on Srivastava-Owa fractional operators. The discrete approximations derived from the Grünwald-Letnikov fractional derivatives present several limitations [28] as follows.

(i) Unstable numerical methods are frequently generated.

(ii) The order of accuracy is never higher than one.

(2) The algorithm of $\mathrm{Pu}$ et al. [23] uses only one FD parameter. By contrast, a key contribution of our work is the multi-FD model. Changing the value of the fractional powers allows the FD coefficients to be adjusted for each image according to various characteristics.

The present study and the study of $\mathrm{Pu}$ et al. [23] have the following characteristics.

(i) The contrast of GLCM increases with increasing $\alpha$. An increasing $\alpha$ corresponds to more pixels, greater contrast, and improved image clarity.

(ii) The correlation of GLCM decreases with increasing $\alpha$, and texture details are less observable.

(iii) The energy of GLCM decreases with increasing $\alpha$, and texture details become less noticeable.

(iv) The homogeneity of GLCM decreases with increasing $\alpha$. Thus, intensive textures are increased. 


\section{Conclusion}

A texture enhancement technique for medical images is introduced by using FD masks based on Srivastava-Owa fractional operators. In the FD spectra, the texture and lightness of the image are enhanced by the FD mask-based algorithm. The proposed algorithm controls the degree of texture enhancement by using the fractional power parameters of the multi-FD model. Our proposed technique is not only limited to medical images but can also be applied to different image applications by considering the limitations of each imaging method. We aim to prevent the effect of noise and control the degree of texture enhancement by using filter mask parameters. The experiment results demonstrate the efficacy of the proposed algorithm.

\section{Acknowledgments}

The authors would like to thank the reviewers for their comments on earlier versions of this paper. This research has been funded by university of Malaya, under RG 100-12 ICT.

\section{References}

[1] R. Hilfer, Applications of Fractional Calculus in Physics, vol. 463, World Scientific, Singapore, 2000.

[2] M. Kanbur, I. Narin, E. Özdemir, E. Dinç, and D. Baleanu, "Fractional wavelet transform for the quantitative spectral analysis of two-component system," in New Trends in Nanotechnology and Fractional Calculus Applications, pp. 321-331, Springer, New York, NY, USA, 2010.

[3] D. Baleanu, K. Diethelm, E. Scalas, and J. J. Trujillo, Fractional Calculus Models and Numerical Methods, vol. 3 of Series on Complexity, Nonlinearity and Chaos, World Scientific, Hackensack, NJ, USA, 2012.

[4] E. Dinç and D. Baleanu, "Ultra-performance liquid chromatography for the multicomponent analysis of a ternary mixture containing thiamine, pyridoxine, and lidocaine in ampules," Journal of AOAC International, vol. 95, pp. 903-912, 2010.

[5] E. Dinç and D. Baleanu, "A new fractional wavelet approach for the simultaneous determination of ampicillin sodium and sulbactam sodium in a binary mixture," Spectrochimica Acta A, vol. 63, no. 3, pp. 631-638, 2006.

[6] H. A. Jalab and R. W. Ibrahim, "Denoising algorithm based on generalized fractional integral operator with two parameters," Discrete Dynamics in Nature and Society, vol. 2012, Article ID 529849, 14 pages, 2012.

[7] A. C. Sparavigna, "Using fractional differentiation in astronomy," Computer Vision and Pattern Recognition, http://arxiv .org/abs/0910.2381v3.

[8] R. Marazzato and A. C. Sparavigna, "Astronomical image processing based on fractional calculus: the AstroFracTool," Instrumentation and Methods for Astrophysics, http://arxiv.org/ abs/0910.4637v2.

[9] Y.-F. Pu, X. Yuan, K. Liao, Z.-L. Chen, and J.-L. Zhou, "Five numerical algorithms of fractional calculus applied in modern signal analyzing and processing," Journal of Sichuan University (Engineering Science Edition), vol. 37, no. 5, article 118, 2005.

[10] Y. Pu, W. Wang, J. Zhou, Y. Wang, and H. Jia, "Fractional differential approach to detecting textural features of digital image and its fractional differential filter implementation," Science in China F, vol. 51, no. 9, pp. 1319-1339, 2008.

[11] T. Glatard, J. Montagnat, and I. E. Magnin, "Texture based medical image indexing and retrieval: application to cardiac imaging," in Proceedings of the 6th ACM SIGMM International Workshop on Multimedia Information Retrieval (MIR '04), pp. 135-142, New York, NY, USA, October 2004.

[12] K. Lu, N. He, and L. Li, "Nonlocal means-based denoising for medical images," Computational and Mathematical Methods in Medicine, vol. 2012, Article ID 438617, 7 pages, 2012.

[13] M. N. Kohan and H. Behnam, "Denoising medical images using calculus of variations," Journal of Medical Signals and Sensors, vol. 1, pp. 184-190, 2011.

[14] F. Luisier, T. Blu, and M. Unser, "A new SURE approach to image denoising: interscale orthonormal wavelet thresholding," IEEE Transactions on Image Processing, vol. 16, no. 3, pp. 593-606, 2007.

[15] H. A. Jalab and R. W. Ibrahim, "Texture feature extraction based on fractional mask convolution with cesáro means for content-based image retrieval," in Proceedings of the 12th Pacific Rim International Conference on Trends in Artificial Intelligence (PRICAI '12), pp. 170-179, 2012.

[16] G. Castellano, L. Bonilha, L. M. Li, and F. Cendes, "Texture analysis of medical images," Clinical Radiology, vol. 59, no. 12, pp. 1061-1069, 2004.

[17] J. R. Smith and S. F. Chang, "Automated binary texture feature sets for image retrieval," in Proceedings of the IEEE International Conference on Acoustics, Speech, and Signal Processing (ICASSP '96), pp. 2239-2242, May 1996.

[18] H. A. Jalab and R. W. Ibrahim, "Texture enhancement based on the savitzky-golay fractional, differential operator," Mathematical Problems in Engineering, vol. 2013, Article ID 149289, 8 pages, 2013.

[19] A. McAndrew, An Introduction to Digital Image Processing with Matlab Notes for SCM2511 Image Processing, School of Computer Science and Mathematics; Victoria University of Technology, 2004.

[20] C. Qing-li, H. Guo, and Z. Xiu-qiong, "A fractional differential approach to low contrast image enhancement," International Journal of Knowledge and Language Processing, vol. 3, pp. 2029, 2012.

[21] Z. Yang, F. Lang, X. Yu, and Y. Zhang, "The construction of fractional differential gradient operator," Journal of Computational Information Systems, vol. 7, pp. 4328-4342, 2011.

[22] V. Garg and K. Singh, "An improved Grunwald-Letnikov fractional differential mask for image texture enhancement," International Journal, vol. 3, no. 3, pp. 130-135, 2012.

[23] Y.-F. Pu, J.-L. Zhou, and X. Yuan, "Fractional differential mask: a fractional differential-based approach for multiscale texture enhancement," IEEE Transactions on Image Processing, vol. 19, no. 2, pp. 491-511, 2010.

[24] R. W. Ibrahim, "On generalized Srivastava-Owa fractional operators in the unit disk," Advances in Difference Equations, vol. 2011, article 55, 10 pages, 2011.

[25] Y. Zhang, Y. Pu, and J. Zhou, "Construction of fractional differential masks based on Riemann-Liouville definition," Journal of Computational Information Systems, vol. 6, no. 10, pp. 3191-3199, 2010.

[26] “MathWorks, Inc, 1984-2009".

[27] R. M. Haralick, K. Shanmugam, and I. Dinstein, "Textural features for image classification," IEEE Transactions on Systems, Man and Cybernetics, vol. 3, no. 6, pp. 610-621, 1973. 
[28] E. Sousa, "How to approximate the fractional derivative of order $1<\alpha \leq 2$, International Journal of Bifurcation and Chaos in Applied Sciences and Engineering, vol. 22, no. 4, Article ID 1250075, 13 pages, 2012. 


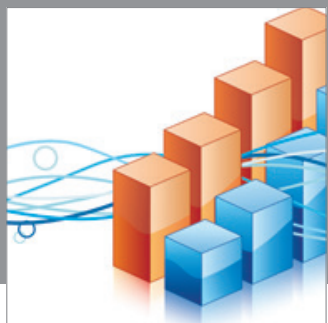

Advances in

Operations Research

mansans

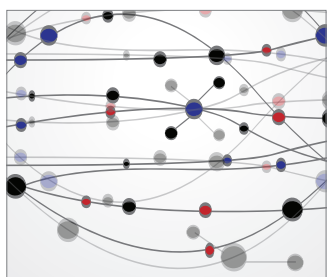

The Scientific World Journal
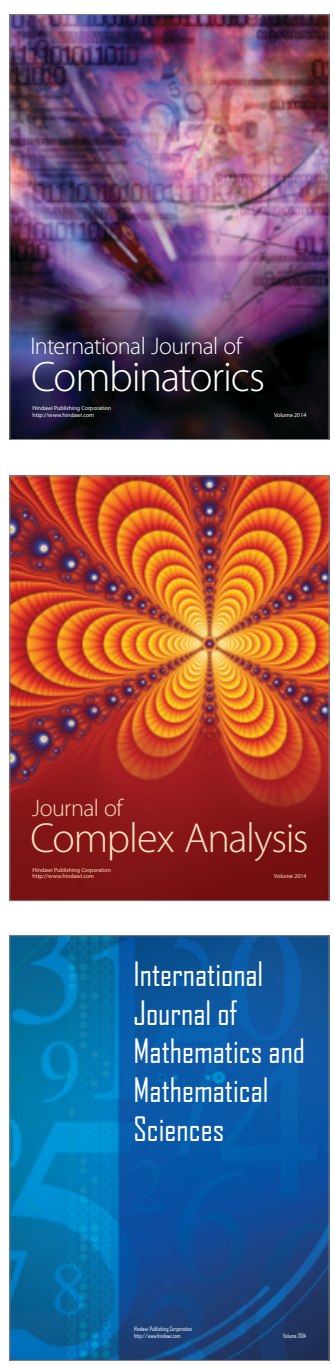
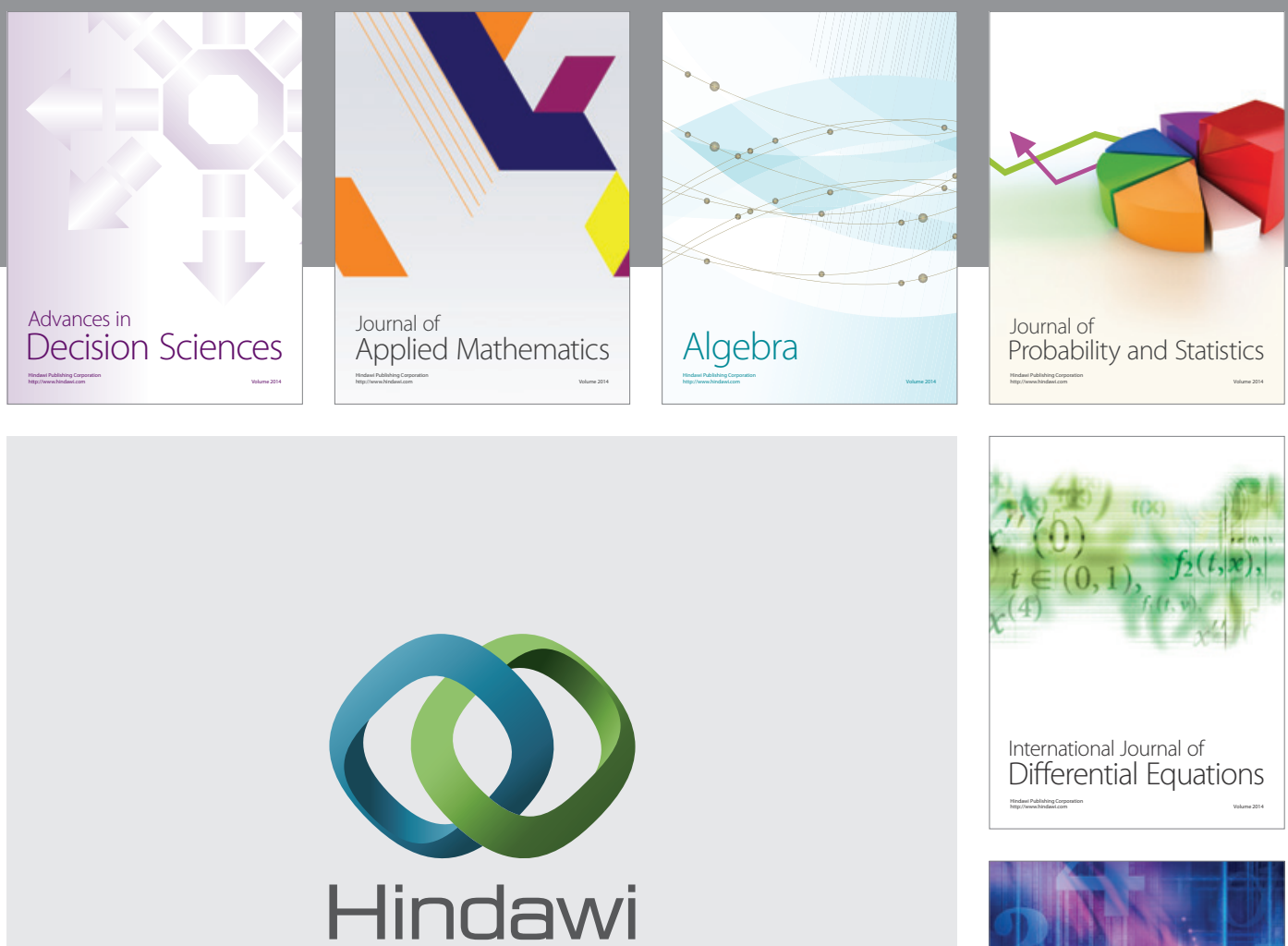

Submit your manuscripts at http://www.hindawi.com
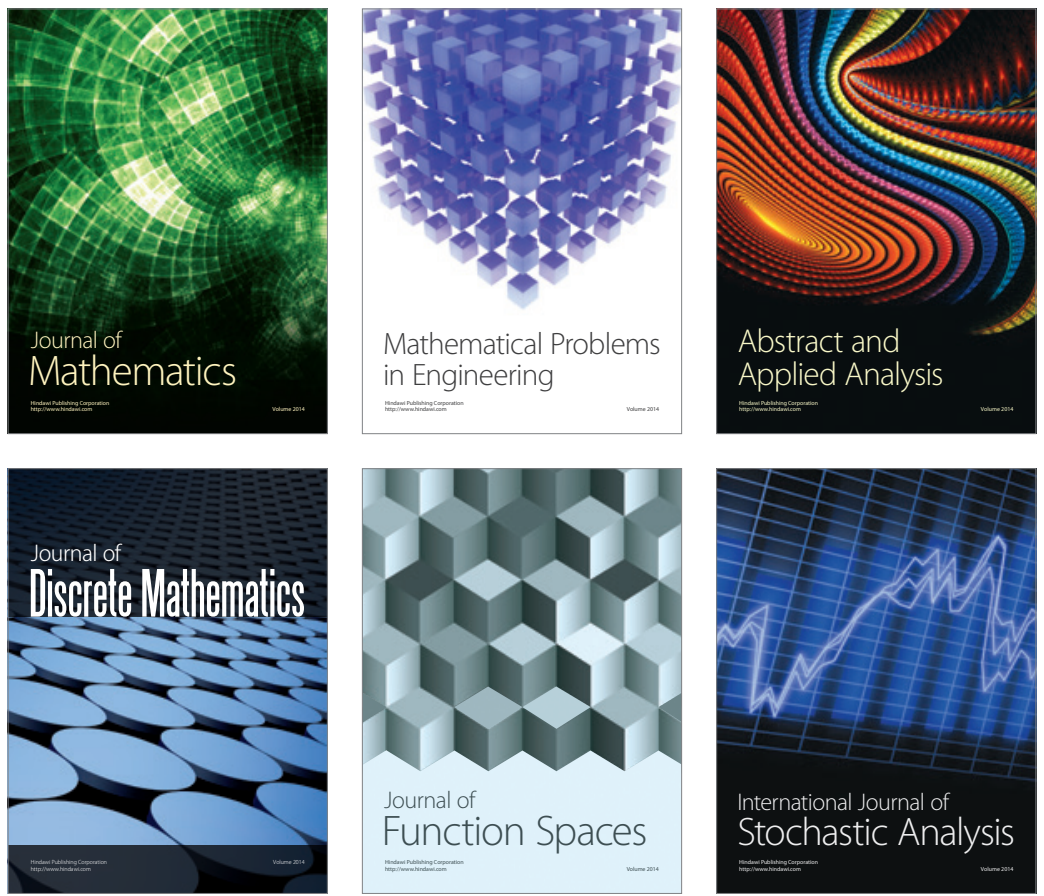

Journal of

Function Spaces

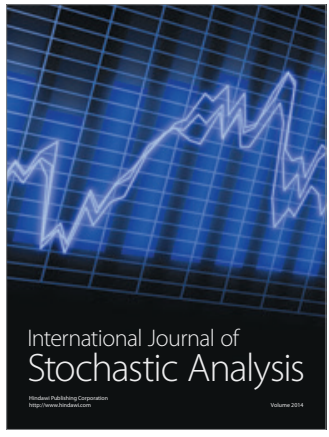

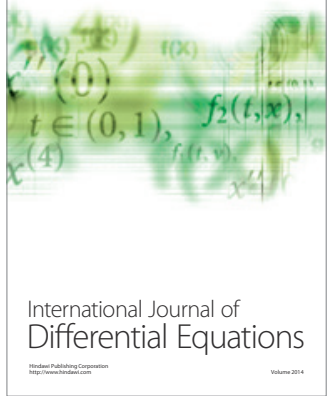
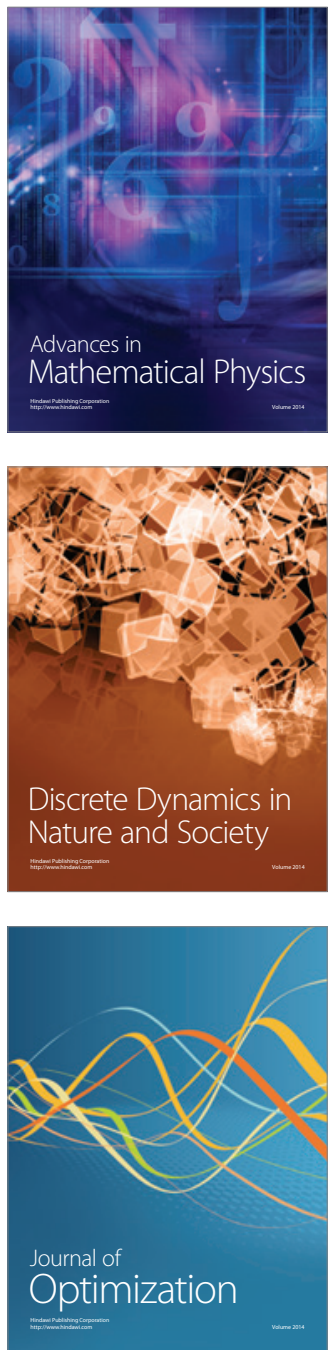